\title{
Persistence and change in the soft X-ray spectrum of the quasar PG $1211+143$
}

\section{Citation}

Elvis, Martin, Belinda J. Wilkes, P. Giommi, and Jonathan McDowell. 1991. "Persistence and Change in the Soft X-Ray Spectrum of the Quasar PG $1211+143$." The Astrophysical Journal 378 (September): 537. doi:10.1086/170454.

\section{Published Version}

10.1086/170454

\section{Permanent link}

http://nrs.harvard.edu/urn-3:HUL.InstRepos:32955263

\section{Terms of Use}

This article was downloaded from Harvard University's DASH repository, and is made available under the terms and conditions applicable to Other Posted Material, as set forth at http:// nrs.harvard.edu/urn-3:HUL.InstRepos:dash.current.terms-of-use\#LAA

\section{Share Your Story}

The Harvard community has made this article openly available.

Please share how this access benefits you. Submit a story.

\section{Accessibility}




\title{
PERSISTENCE AND CHANGE IN THE SOFT X-RAY SPECTRUM OF THE QUASAR PG
}

$$
1211+143
$$

\author{
MARTIN ELVIS \\ Harvard-Smithsonian Center for Astrophysics, 60 Garden Street, Cambridge, MA 02138 \\ P. GIOмM \\ ESTEC, EXOSAT Observatory, Keplerlaan 1, 2200 AG Noordwijk, The Netherlands \\ AND \\ BELINDA J. WILKeS AND JONATHAN MCDOWELl ${ }^{1}$ \\ Harvard-Smithsonian Center for Astrophysics, 60 Garden Street, Cambridge, MA 02138 \\ Received 1990 December 20; accepted 1991 March 18
}

\begin{abstract}
Two Einstein and three EXOSAT observations of PG $1211+143$ over a 6 year baseline show that strong steep-spectrum low-energy $\mathrm{X}$-ray emission is a persistent feature of this quasar. EXOSAT observations of PG $1211+143$ detected a factor of 2.3 increase in its steep soft X-ray (C-band) flux in 18 days. The bulk of the soft $X$-ray emission of $P G 1211+143$ therefore comes from a region less than $5 \times 10^{16} \mathrm{~cm}$ across. In another (quasar rest frame) interval of 193 days the soft X-rays decreased by a factor of 3.7. Either an intrinsic emission variation or an increase of $N_{H}$ from 3.4 to $7.6 \times 10^{20}$ atoms $\mathrm{cm}^{-2}$ could produce the same change. In the same time interval the hard $(2-10 \mathrm{keV}) \mathrm{X}$-rays decreased by a factor $1.6 \pm 0.05$. This suggests a connection between the two energy regimes and argues against variable absorption causing the soft $\mathrm{X}$-ray variations. In order to decrease in luminosity so rapidly a thermal source in PG $1211+143$ would have to be optically thick to both electron scattering and free-bound absorption. The same argument applies to six of the seven other rapidly variable soft excesses reported in the literature, which suggests that optically thin thermal models are ruled out in general.
\end{abstract}

Subject headings: quasars - X-rays: sources - X-rays: spectra

\section{INTRODUCTION}

"Soft X-ray excesses" are a new component of the quasar continuum. At energies below the carbon edge at $0.28 \mathrm{keV}(\geq 44 \AA$, the "C-band") half of all studied quasars and AGNs are dominated by large excess flux over an extrapolation of their higher energy power laws (Arnaud et al. 1985; Wilkes \& Elvis 1987; Turner \& Pounds 1989; Turner et al. 1990; Masnou et al. 1991; Comastri et al. 1991 $)^{2}$. It has been suggested that these soft X-ray/extreme-ultraviolet excesses are emitted from the inner edges of accretion disks (Arnaud et al. 1985; Bechtold et al. 1987; Czerny \& Elvis 1987). In this case their radiation comes to us from the innermost identifiable region of a quasar. The spectral shapes of the soft $\mathrm{X}$-ray excesses might then give us information on the peak temperatures in disks and hence determine the allowed values of the central masses and accretion rates.

Whatever their origin this new component deserves careful investigation. Existing constraints on their spectra are poor because the X-ray measurements cover a relatively small energy range $(\sim 0.1-0.3 \mathrm{keV})$ and have almost no energy resolution. Also very few objects were observed more than once so that variability information is scarce. Clearly more studies of these ultrasoft excesses are needed. Variability data in particular will be most important to distinguish between extended and compact emission regions, and to limit the properties of accretion disk models (Siemiginowska \& Czerny 1989).

PG $1211+143$ is an extreme case of a quasar with a soft X-ray excess. It has an excess extending to higher energies than in other cases ( $\sim 2 \mathrm{keV}$; Bechtold et al. 1987), and is the only one of the 33 quasars with soft X-ray spectra in the Einstein IPC survey to be dominated by an extremely steep, soft X-ray spectrum (Wilkes \& Elvis 1987). We therefore undertook a program of X-ray observations of PG $1211+143$ with the EXOSAT Observatory. This paper reports this series of observations.

The value of the absorbing Galactic column density is crucial to studies of soft X-ray excesses (see, e.g., Elvis, Wilkes, \& McDowell 1990). We use the accurate Galactic column density of $2.8 \pm 0.1 \times 10^{20}$ atoms cm ${ }^{-2}$ from the study by Elvis, Lockman, \& Wilkes (1989). Values of the Hubble constant $H_{0}=50 \mathrm{~km} \mathrm{~s}^{-1} \mathrm{Mpc}^{-1}$ and of $q_{0}=0$ are used throughout. The redshift of PG $1211+143$ is 0.085 (Schmidt \& Green 1983).

\section{OBSERVATIONS}

PG $1211+143$ was observed on three separate occasions by EXOSAT with intervals of 209 and 19 days between the observations. (For details of the EXOSAT instrumentation see de Korte et al. 1981 and Turner, Smith, \& Zimmerman 1981). Table 1 gives details of the observations, including the derived count rates.

\footnotetext{
1 Present address: NASA/Marshall Space Flight Center, ES-65 Marshall Space Center, AL 35801.

2 In fact their very soft spectra suggest that they would be more accurately named “XUV excesses" to denote the energy band below the carbon edge; however, common usage has settled on the less precise "soft X-ray excess" which could apply to any energy from $\sim 0.2$ to $2 \mathrm{keV}$.
} 
TABLE 1

EINSTEIN AND EXosat OBSERVATIONS OF PG $1211+143$

\begin{tabular}{cccc}
\hline \hline & & \multicolumn{3}{c}{ Exposure } \\
Year/Day & Instrument 'Filter & $(\mathrm{s})$ & Count per second \\
\hline $1979 / 339$ & IPC & 6306 & $0.89 \pm 0.02$ \\
$1980 / 347$ & IPC & 1795 & $1.28 \pm 0.03$ \\
$1985 / 164$ & ME $^{2}$ & 22370 & $0.84 \pm 0.02$ \\
& LE/3LX & 1257 & $0.206 \pm 0.012$ \\
& LE/ALP & 3539 & $0.0867 \pm 0.006$ \\
& LE/BOR & 16560 & $0.0087 \pm 0.0012$ \\
$1986 / 008$ & ME & 17030 & $0.52 \pm 0.02$ \\
& LE/3LX & 15753 & $0.055 \pm 0.0025$ \\
$1986 / 028$ & LE/3LX & 10065 & $0.13 \pm 0.004$ \\
& LE/ALP & 15753 & $0.05 \pm 0.009$ \\
\hline
\end{tabular}

PHA 7-40, 2-10 keV.

The quasar was seen in all of the three energy bands defined by the "thin lexan" (3LX), "aluminum/parylene" (ALP) and "boron" (BOR) filters, which each cover the $\sim 0.1-2 \mathrm{keV}$ band with somewhat differently weighted responses, in front of the LE giving smaller effective artiplier array) during the first observation. In the later observations the source was fainter so the filters $1211+143$.

The positions of the source in all the LE detections are consistent with one another and with the optical (Schmidt \& Green 1983) position of PG $1211+143$ to within the 6" uncertainties of the EXOSAT aspect (Osborne \& Angelini 1986). The LE source appears pointlike in all the exposures. The mean LE position for the source is: $\alpha=12^{\mathrm{h}} 11^{\mathrm{m}} 44^{\mathrm{s}} .9 \pm 0.4 ; \delta=14^{\circ} 19^{\prime} 53^{\prime \prime} \pm 6^{\prime \prime}(1950.0)$

The LE count rates (Table 1) were estimated using a square box centered on the source centroid and of a size which maximizes the to take into account spatial nonuniformities in the from a nearby source-free area of the image and rescaled to the source position outside the box

(D) box (Davelaar \& Giommi 1985), for vignetting effects, and for telemetry and instrumental dead times (Osborne 1985).

subtraction in the thirdions the source was clearly detected in the ME (medium energy) argon detectors. Poor background much noisier than " therefore used only the "outer" method of Smith (1984). The background in each case was taken from subtraction for the ME was performed according to the from the source during " arackground in each case was taken from the same detectors during times when they were pointed away curves of A serendipitous observation

Counter, 0.2-3.5 keV; Giacconi PG 1211+ 143 (sequence number 6982) was made by the Einstein IPC (Imaging Proportional year earlier than the off-axis. At such positions the gain ("BAL" which measures the conversion from recordine. In this observation the quasar was 21 '.8 must be estimated using the conversion from recorded pulse height to incoming photon energy) $\sim 12$ to 19). The IPC count rate response function. The targeted Einstein IPC observation vignetting and the loss of counts due to the degraded off-axis point Bechtold et al. (1987), and Wilkes \& Elvis (1987), had a $20 \%$ higher count rate.

\section{PERSISTENCE: A 6 YEAR SOFT EXCESS}

The unusual feature of PG $1211+143$ is its steep soft X-ray spectrum. It is important to understanding this emission to know whether it is persistent or transient. The five EXOSAT LE and Einstein observations allow us to test this.

We have fitted a simple power-law plus absorption model ${ }^{3}$ to each of the data sets. Table 2 gives the results of these fits. (The spectrum from the first EXOSAT observation is reported by Comastri et al. 1991). The errors on each fit are quite large, but the error regions overlap in both spectral parameters. It is notable that the well-constrained fits (i.e., excluding the $79 / 339$ and $86 / 008$ data) give steeper values for the power-law index than are typical of either low-energy quasar spectra (Wilkes \& Elvis 1987), or of higher energy AGN spectra (Mushotzky 1984; Turner \& Pounds 1989). When the Galactic column density is imposed as a minimum value then slopes of around $\alpha_{E} \sim 2.0$ are required, even for the 79/339 (off-axis IPC) data. However, the $\chi^{2}$ values even for the best fit values (Table 2) are not good, which suggests that the spectrum has curvature, steepening toward lower energies.

The LE data for 1985/164, having three data points, allow a fit to be made. This fit suggests an even steeper slope, $\sim 3.0$. A blackbody fit to the same 1985/164 LE data gives a best-fit temperature of $75 \pm 5 \mathrm{eV}$.

The unusually steep spectrum of PG $1211+143$ is thus not a transitory feature but is seen in observations spanning 6 years.

${ }^{3} f_{v} \propto v^{-\alpha_{\varepsilon}} e^{-N_{H \sigma}}$, cross sections $(\sigma)$ were taken from Morrison \& McCammon (1983). 
TABLE 2

Power-Law Fits to X-Ray Data For PG $1211+143$

\begin{tabular}{clccccc}
\hline \hline Year/Day & Instrument(s) & $\alpha_{E}^{\mathrm{a}}$ & $N_{\mathbf{H}}^{\mathrm{a}, \mathrm{b}}$ & Norm $^{\mathrm{a}, \mathrm{c}}$ & $\chi^{2}$, d.o.f. & $P\left(\chi^{2}\right)^{\mathrm{e}}$ \\
\hline $1979 / 339 \ldots \ldots \ldots$ & IPC $^{\mathrm{f}, \mathrm{g}}$ & $1.3_{-0.5}^{+0.9}$ & $0.9_{-0.9}^{+1.5}$ & $4_{-1}^{+46}$ & 12,5 & 0.04 \\
$1980 / 346 \ldots \ldots \ldots$ & IPC $^{\mathrm{g}}$ & $2.0_{-0.7}^{+1.7}$ & $2.3_{-0.6}^{+4.8}$ & $5.5_{-0.7}^{+0.8}$ & 12,5 & 0.04 \\
$1985 / 164 \ldots \ldots \ldots$ & $\mathrm{ME}^{\mathrm{h}}$ & $1.7_{-0.4}^{+0.6}$ & $0_{-0}^{+85}$ & $4.4_{-1.3}^{+4.8}$ & 48,31 & 0.08 \\
$1985 / 164 \ldots \ldots \ldots$ & $\mathrm{ME}^{\mathrm{h}}+\mathrm{LE}$ & $1.8_{-0.2}^{+0.3}$ & $2.2_{-0.9}^{+1.5}$ & $4.8_{-1.0}^{+1.3}$ & 56,34 & 0.01 \\
$1985 / 164 \ldots \ldots \ldots$ & $\mathrm{LE}$ & $2.9_{-0.9}^{+2.4}$ & $3.4_{-1.5}^{+6.5}$ & $2.4_{-2.1}^{+2.7}$ & 0,0 & $\ldots$ \\
$1986 / 008 \ldots \ldots \ldots$ & $\mathrm{ME}^{\mathrm{h}}$ & $0.9_{-0.6}^{+1.2}$ & $0.0_{-0.0}^{+3.0}$ & $1.1_{-0.6}^{+5.5}$ & 47,31 & 0.04 \\
$1986 / 008 \ldots \ldots \ldots$ & $\mathrm{ME}^{\mathrm{h}}+\mathrm{LE}$ & $0.9_{-0.4}^{+0.7}$ & $0.3_{-0.3}^{+2.5}$ & $1.1_{-0.4}^{+1.3}$ & 47,32 & 0.04 \\
\hline
\end{tabular}

${ }^{a}$ Ninety percent confidence ranges for three interesting parameters $\left(\chi_{\min }^{2}+6.25 ;\right.$ Avni 1976$)$

b $10^{20}$ atom $\mathrm{cm}^{-2}$

c Microjanskys at $1 \mathrm{keV}$.

d Degrees of freedom.

e Probability of obtaining the observed $\chi^{2}$.

${ }^{\text {f }}$ PHA 2-9.

${ }^{8}$ Errors include IPC gain uncertainties at this off-axis position.

${ }^{\text {h }}$ PHA $7-40$.

\section{CHANGE: OPTICALLY THICK THERMAL EMISSION}

On time scales of days to months significant variability is seen between each of the EXOSAT observations. In particular, in the quasar's frame, the LE 3LX data show a factor of $3.7 \pm 0.3$ decrease in 193 days and a factor $2.3 \pm 0.1$ increase in 18 days (Table 1 ). In the 193 day interval the hard (2-10 keV) X-rays also decreased, although only by a factor $1.6 \pm 0.05$. This may indicate some physical link between the emission in the two energy regimes.

The variations may be due either to intrinsic emission variability or to changing intervening absorption. At these low energies quite small column densities can produce such changes. To produce the decrease by a factor of 3.7 in the $3 \mathrm{LX}$ filter seen between the first (1985 June) and second (1986 January) EXOSAT observations needs an increase in $N_{\mathrm{H}}$ from $3.4 \times 10^{20}$ atoms $\mathrm{cm}^{-2}$ (the best-fit value for the first observation) to $7.6 \times 10^{20}$ atoms $\mathrm{cm}^{-2}$. This is a minimum value. Material with a larger column density could obscure up to half the source to produce the same effect.

The contemporaneous decrease in the higher energy ME count rate by a factor of 1.6 argues against absorption as the only variable since $2-10 \mathrm{keV} X$-rays are negligibly affected by column densities less than $10^{21}$ atoms $\mathrm{cm}^{-2}$.

If the XUV excess were due to an optically thin thermal plasma then its spectrum would be rich in diagnostic emission lines for the inner regions of quasars (Marshall 1991). However, the variability information rules out an optically thin plasma quite strongly, assuming an intrinsic change in the emission, since it requires large optical depths to both bound-free and electron scattering in the emitting plasma, as shown below.

If we take the decrease in $3 \mathrm{LX}$ count rate between the first and second EXOSAT observations to define a minimum cooling time, we can derive an optical depth for the emission region, taking into account electron scattering within the source, which smears out variations and increases the observed time scales by a factor $\left(1+\tau_{\text {es }}\right)$. Of course more rapid variations may have taken place between our observations, but the shorter implied cooling times only increase the required optical depth derived below. For simplicity we consider a uniform density sphere of constant temperature plasma.

A spherical optically thin source of radius $r$ with electron density $n=\tau_{\text {es }} / r \sigma_{\text {es }}$ and temperature $T$ has luminosity in the $0.1-0.3 \mathrm{keV}$ range of

$$
L=\frac{4}{3} \pi r^{3} F_{0} f n^{2} T^{1 / 2} B(T)=\left[\frac{4}{3} \pi \tau_{\mathrm{es}}^{2} \sigma_{\mathrm{es}}^{-2} F_{0} f T^{1 / 2} \exp \left(-\frac{0.1 \mathrm{keV}}{k T}\right)\right] r
$$

where

the bandpass factor

$$
F_{0}=2.4 \times 10^{-27} \mathrm{ergs} \mathrm{cm}^{3} \mathrm{~K}^{-1 / 2} \mathrm{~s}^{-1}
$$

$$
B(T)=\exp \left(-\frac{0.1 \mathrm{keV}}{k T}\right),
$$

which is sensitive to the uncertain effective lower frequency limit of the soft excess observation, and $f$ is a factor giving the additional contribution of recombination and line emission to bremsstrahlung cooling, $1<f<10$ (Raymond \& Smith 1977; Raymond 1990). The radius in this equation can be related to the time scale set by the variability, $t_{\mathrm{var}}$ by

$$
r \leq \frac{c t_{\mathrm{var}}}{\left(1+\tau_{\mathrm{es}}\right)}
$$

(optically thick sources vary slowly for their size, so the inferred size is smaller for high depth), and so, combining the above equations,

$$
\frac{\tau_{\mathrm{es}}^{2}}{1+\tau_{\mathrm{es}}} \geq g
$$


where

$$
\begin{aligned}
g & =\frac{3 L \sigma_{\mathrm{T}}^{2}}{4 \pi c t_{\mathrm{var}} F_{0} f T^{1 / 2} B(T)}=1.4 \times 10^{-33}\left(\frac{L}{1 \mathrm{ergs} \mathrm{s}^{-1}}\right) f^{-1}\left(\frac{t_{\mathrm{var}}}{1 \mathrm{~s}}\right)^{-1}(T / 1 \mathrm{~K})^{-1 / 2} \exp \left[-1.2\left(\frac{T}{1 \mathrm{~K}}\right)^{-1}\right] \\
& =7.1 \times 10^{2}\left(\frac{L}{10^{44} \mathrm{ergs} \mathrm{s}^{-1}}\right) f^{-1}\left(\frac{t_{\mathrm{var}}}{1 \mathrm{day}}\right)^{-1}\left(\frac{T}{5 \times 10^{6} \mathrm{~K}}\right)^{-1 / 2} \exp \left[-0.24\left(\frac{T}{5 \times 10^{6} \mathrm{~K}}\right)^{-1}\right] .
\end{aligned}
$$

If $g<1$, it is the square of the electron scattering depth. If $g>1$, the assumption of optical thinness leads to a contradiction, and we may deduce that the source must be optically thick; $g$ is then equal to the electron scattering depth as long as the formula for $L$ remains valid.

Even if the source is optically thick to electron scattering, it will retain its optically thin spectral shape unless absorptive opacity is also present. Bound-free interactions are dominant in modifying the spectrum toward a blackbody. The bound-free optical depth $\tau_{\mathrm{bf}}$ is just $\tau_{\mathrm{es}}\left(\sigma_{\mathrm{bf}} / \sigma_{\mathrm{es}}\right)$ (where the $\sigma$ are the corresponding cross sections). The bound-free cross section at these subkilovolt energies depends only weakly on temperature in the range above $10^{6} \mathrm{~K} ; \sigma_{\mathrm{bf}} / \sigma_{\mathrm{es}}$ at $0.1-0.2 \mathrm{keV}$ is in the range $1-5$ with $T$ between $10^{6}$ and $10^{7}$ K (Krolik \& Kallman 1984; Kallman private communication) and so the spectrum will indeed be modified toward thermal form if the source is optically thick. The upper limit to the optical depth obtainable from comparing the blackbody radius to the variability time scale is not interesting.

The decrease in $3 \mathrm{LX}$ count rate between the first and second EXOSAT observations of PG $1211+143$ gives a value $g$ of $\sim 14$ $(f=1)$, which implies that it is optically thick. If instead the flux decrease is due to changing absorption in front of the source then electron scattering delays do not apply. The variability gives the physical size directly if we assume an absorber local to the quasar which moves to occult at least the fraction of the source whose luminosity disappears. For a transverse velocity of the absorber, $v$, the optical depth to electron scattering is then $\sim 180(\mathrm{v} / \mathrm{c})^{-1 / 2}$, which is considerable even for a relativistically moving intervening absorber.

Variability of soft X-ray excesses is now known to be common in quasars and AGNs (see summary in Elvis, Wilkes, \& McDowell 1991). In some cases the variations are far more rapid than in PG $1211+143$ (e.g., Mrk 335; Turner \& Pounds 1988 ; Lee \& Balick 1988). Table 3 lists the seven cases in the literature for which luminosities and time scales for luminosity decreases are given. (Only decreases in luminosity were used since only these define cooling times.) These variations imply the values of $g$ listed in Table 3 and are shown in Figure 1 against lines showing the $g=0.5(\tau=1)$ and $g=10$ loci for electron scattering.

PG $1211+143$ is seen from Figure 1 (and Table 3) to be one of the least extreme of these soft X-ray excesses. Most are factors of 10-100 times more optically thick, to the extent that large values of $g$ imply a large optical depth. (E1615 +061 is the only exception but here the excess may not be real; Buckley et al. 1987). We conclude that optically thin emission rarely dominates the production of quasar soft X-ray excesses, so that the line emission will be broadened into a continuum. Optically thick emission would tend to support an origin in the central regions of an accretion disk, although we note that the physics of the inner edge are not well understood (Shakura \& Sunyaev 1973), and that other processes (e.g., Comptonization; Czerny \& Elvis 1987) could well modify the emitted disk spectrum.

The changes in the LE/3LX flux of PG $1211+143$ between the EXOSAT observations trivially rule out a source for the soft excess X-rays in extended regions on the scale of the optical/ultraviolet narrow emission line cloud zones ( $\sim 100 \mathrm{pc}-1 \mathrm{kpc})$, such as suggested by Halpern \& Filippenko (1984) and observed in several Seyfert galaxies (Elvis et al. 1991). They would also have ruled

TABLE 3

\begin{tabular}{|c|c|c|c|c|c|}
\hline Name & $\begin{array}{c}\log L_{\mathbf{x s}^{a}}^{a} \\
\left(\operatorname{ergs~s}^{-1}\right)\end{array}$ & $\underset{(\mathrm{s})}{\log \Delta t}$ & $\begin{array}{c}\text { Variability } \\
\text { factor }\end{array}$ & $g^{\mathrm{b}}$ & Reference \\
\hline Mrk $335 \ldots \ldots \ldots \ldots$ & 43.6 & 4.3 & 2.0 & 1000 & 1,2 \\
\hline Fairall $9 \ldots \ldots \ldots \ldots$ & 44.9 & 5.7 & 1.9 & 450 & 3 \\
\hline M81 ................ & 40.5 & 2.7 & 2.0 & 30 & 4 \\
\hline $3 \mathrm{C} 273 \ldots \ldots \ldots \ldots$ & 45.2 & 7.2 & 1.7 & 65 & 5,6 \\
\hline NGC $4051^{c} \ldots \ldots \ldots$ & 41.5 & 3.0 & 2.0 & 300 & 7 \\
\hline PG $1211+143 \ldots \ldots$. & 44.7 & 7.2 & 3.7 & 14 & 8 \\
\hline NGC $5548 \ldots \ldots \ldots$ & 44.6 & 4.4 & 1.3 & 8100 & 9 \\
\hline Mrk $841 \ldots \ldots \ldots \ldots$ & 44.4 & 4.6 & 1.5 & 3200 & 10 \\
\hline $\mathrm{E} 1615+061^{\mathrm{d}} \ldots \ldots \ldots$ & 44.0 & 8.0 & 1.5 & 0.5 & 11 \\
\hline
\end{tabular}

OPTICAL DePthS for VARIABLE SOFt EXCESSES IN AGN

a Luminosity in the soft excess, $\sim 0.1-0.3 \mathrm{keV}$

${ }^{b}$ For $T=5 \times 10^{6} \mathrm{~K}, f=1$. Values are $\sim 5$ times smaller for $T=1 \times 10^{6} \mathrm{~K}$, while $\tau_{\mathrm{bf}}$ is $\sim 85$ times larger.

c Note the warnings of Lawrence et al. (1987) about picking time scales from $1 / \mathrm{f}$ variations.

d But see Buckley et al. (1987) who note a nearby X-ray bright RSCVn system. This may be the cause of the apparent soft excess in this AGN.

Reference.--(1) Turner \& Pounds 1988; (2) Lee et al. 1988; (3) Morini et al. 1986; (4) Barr et al. 1985 (quoted in Barr \& Mushotzky 1986); (5) Turner et al. 1990; (6) Masnou et al. 1991; (7) Lawrence et al. 1985; (8) this paper; (9) Kaastra \& Barr 1989; (10) Arnaud et al. $1985 ;$ (11) Pravdo et al. 1981. 


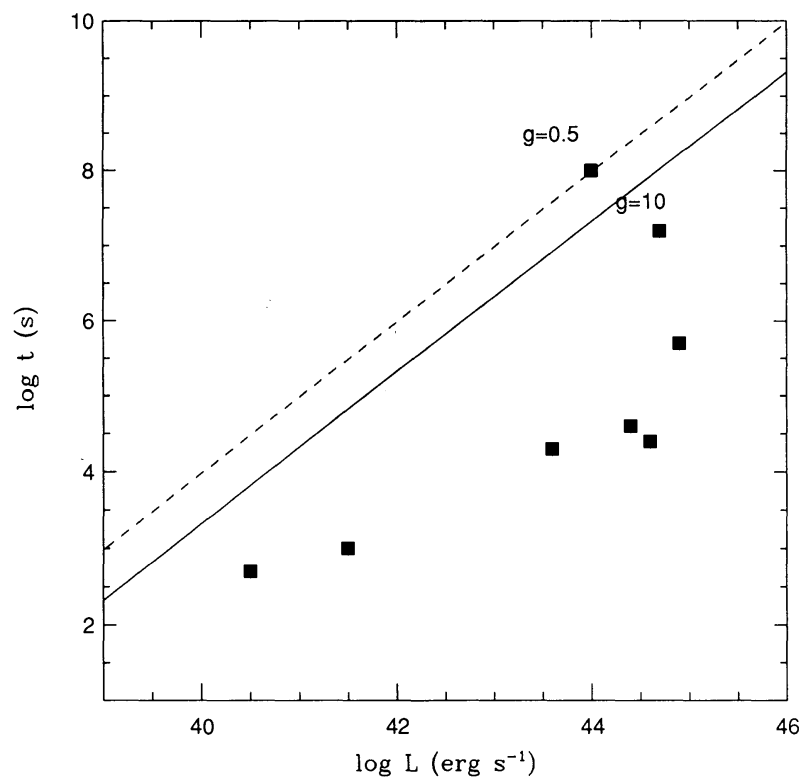

FIG. 1.-Luminosity in the "soft X-ray excess" component vs. observed time scale for a decrease in the soft X-ray component for the AGN from Table 3. The lines show values of $g=1$ (marginally optically thick) and $g=10$ for thermal emission with electron scattering. Larger optical depths lie to the bottom right, which implies that most soft excesses are optically thick.

out emission from the broad emission-line regions (Krolik, McKee, \& Tarter 1981) under earlier assumptions about their size, however, recent observations (Peterson et al. 1990) suggest that sizes of 5-30 light days are quite plausible for these regions, comparable to the light travel time size limits derived for the soft excess emission regions.

\section{CONCLUSIONS}

Over five observations covering 6 years the steep low-energy X-ray spectrum of PG $1211+143$ was always present. In this quasar the "soft excess" is a persistent feature.

EXOSAT observations of the quasar PG $1211+143$ have detected large amplitude (factor $>2$ ) short time-scale changes in its low-energy X-ray emission: an increase in 18 days and a decrease in 193 days. The small size implied for the bulk of the soft X-ray emission from PG 1211+143 implies large optical depths in the emitting plasma. This conclusion is true of most known variable soft X-ray excesses in AGNs and quasars. Optically thick emission is likely from the inner regions of an accretion disk, although modifications (due to, e.g., Comptonization) can occur, and the detailed physics is not well understood.

Observations of variability and spectra of the soft X-ray excess component of AGN continuum emission are proving valuable. ROSAT observations are likely to extend this work substantially.

We thank K. Arnaud, R. Shafer, and A. Tennant for their work on the XANADU data analysis system, J. Lockman for advice on the Galactic column density and to A. Laor and J. Raymond. The IPC data came from the Einstein data bank. M. E. thanks the ESTEC EXOSAT staff for their hospitality and support during a visit for data analysis. This work was supported in part by NASA grant NAG8-568 (EXOSAT), NASA contract NAS8-30751 (HEAO 2), and by NASA Astrophysics Data Program grant NAG8-689.

\section{REFERENCES}

Arnaud, K. A., et al. 1985, MNRAS, 217, 105

Avni, Y. 1976, ApJ, 210, 642

Barr, P., Giommi, P., Wamsteker, W., Gilmozzi, R., \& Mushotzky, R. F. 1985, BAAS, 17 , No. 2,608

Barr, P., \& Mushotzky, R. F. 1986, Nature, 320, 421

Bechtold, J., Czerny, B., Elvis, M., Fabbiano, G., \& Green, R. F. 1987, ApJ, 314, 699

Buckley, D. A. H., Tuohy, I. R., Remillard, R. A., Bradt, H. V., \& Schwartz, D. A. 1987, ApJ, 315, 273

Comastri, A., et al. 1991, ApJ, submitted

Czerny, B., \& Elvis, M. 1987, ApJ, 321, 694

Davelaar, J., \& Giommi, P. 1985, EXOSAT Express, No. 10, 45

de Korte, P. A. J., et al. 1981, Space Sci. Rev., 30, 495

Elvis, M., Fassnacht, C., Wilson, A. S., \& Briel, U. 1991, ApJ, 361, 459

Elvis, M., Lockman, F. J., \& Wilkes, B. J. 1989, AJ, 97, 777

Elvis, M., Wilkes, B. J., \& McDowell, J. C. 1991, in EUV Astronomy, ed.

R. Malina \& S. Bowyer (New York: Pergamon), p. 238

Elvis, M., Wilkes, B. J., \& Tananbaum, H. 1985, ApJ, 292, 357
Giacconi, R., et al. 1979, ApJ, 230, 540

Giommi, P. 1985, EXOSAT Express, No. 12, p. 33

Gorenstein, P., Harnden, R. F., Jr., \& Fabricant, D. 1981, IEEE Trans. Nucl. Sci., NS-28, 869

Halpern, J. P., \& Filippenko, A. V. 1984, ApJ, 285, 475

Harnden, R. F., Jr., Fabricant, D. G., Harris, D. E., \& Schwartz, J. 1984, SAO Spec. Rept. No. 393

Kaastra, J. S., \& Barr, P. 1989, A\&A, 226, 59

Krolik, J. H., \& Kallman, T. 1984, ApJ, 286, 366

Krolik, J. H., McKee, C. F., \& Tarter, C. B. 1981, ApJ, 249, 422

Lawrence, A., Watson, M. G., Pounds, K. A., \& Elvis, M. 1985, MNRAS, 217, 685

. 1987, Nature, 253,694

Lee, M. G., \& Balick, B. 1988, ApJ, 331, 154

Marshall, H. L. 1991, in EUV Astronomy, ed. R. Malina \& S. Bowyer (New York: Pergamon), p. 228

Masnou, J.-L., Wilkes, B. J., Elvis, M., McDowell, J. C., \& Arnaud, K. A. 1991 A\&A, in press 
Morini, M., et al. 1986, ApJ, 307, 366

Morrison, R., \& McCammon, D. 1983, ApJ, 270, 119

Mushotzky, R. F. 1984, Adv. Space Res., 3, Nos. 10-12, 157

Osborne, J. 1985, EXOSAT Express, No. 13, 42

Osborne, J., \& Angelini, L. 1986, EXOSAT Express, No. 17, 3

Peterson, B. M., et al. 1991, ApJ, 368, 119

' Pravdo, S. H., Nugent, J. J., Nousek, J. A., Wilson, A. S., \& Becker, R. H. 1981,

InI ApJ, 251, 501

I.I Raymond, J. 1990, private communication

Raymond, J., \& Smith, B. W. 1977, ApJS, 35, 419
Schmidt, M., \& Green, R. F. 1983, ApJ, 232, 463

Siemiginowska, A., \& Czerny, B. 1989, MNRAS, 239, 289

Shakura, N. I., \& Sunyaev, R. A. 1973, A\&A, 24, 337

Smith, A. 1984, EXOSAT Express, No. 5, 48

Turner, M. J., Smith, A., \& Zimmerman, H. U. 1981, Space Sci. Rev., 30, 513

Turner, T. J., \& Pounds, K. A. 1988, MNRAS, 232, 463

- 1989, MNRAS, 240,833

Turner, M. J., et al. 1990, MNRAS, 244,310

Wilkes, B. J., \& Elvis, M. 1987, ApJ, 323, 243 